\title{
Ocular Infections In School Children In A Rural Block Of Haryana
}

\author{
S Sharma, B Vashisht, M Kalhan, M Goel
}

\section{Citation}

S Sharma, B Vashisht, M Kalhan, M Goel. Ocular Infections In School Children In A Rural Block Of Haryana. The Internet Journal of Epidemiology. 2009 Volume 6 Number 2.

DOI: $\underline{10.5580 / 1 b 57}$

\begin{abstract}
Objectives: To study the prevalence of ocular infections in school children (6-15 years) and their association with age and sex. Study Design: Cross-sectional. Setting: Govt. Senior Secondary Schools of Block Lakhanmajra. Participants: 1265 school children (6-15 years). Methodology: Out of 16 Govt. Senior Secondary Schools, 4 were randomly chosen. Students aged 6-15 years studying in class 1 to 10 were included in the study. The detailed examination included external examination with torch \& 2x magnifying loupe. The finding of clinical examination was recorded on a pretested Performa and were analysed. Statistical Analysis: percentages and Chi-square test. Result: Out of 1265 students examined, $288(22.8 \%)$ were found to have ocular infections. Most common infection was squamous blepharitis (12.3\%) followed by vernal conjunctivitis (5.1\%), conjunctivitis $(4.7 \%)$, stye $(0.7 \%)$. Conclusion: There was high prevalence of ocular infections in students. Health education regarding eye hygiene and eye care should be given to students.
\end{abstract}

\section{INTRODUCTION}

Ocular infections are one of the major causes of ocular morbidity and blindness in India. The rate of infections and complications are influenced by a number of socio-cultural and economic factors. Conjunctivitis is a common problem in our country especially in summer months. Acute conjunctivitis is the most common of all eye infections. Since most conjunctivitis are viral in origin, personal hygiene and care of eyes are most important in prevention and control. Phlyctenular conjunctivitis is especially frequent in the presence of debilitating diseases like Tuberculosis. Vernal conjunctivitis is related with spring season and caused by a hypersensitivity reaction to exogenous allergens $_{1}$.

\section{OBJECTIVES}

To study the prevalence of ocular infections in school children (6-15 years) and their association with age and sex.

\section{MATERIAL AND METHODS}

The present cross-sectional study was carried out from September 2006 to July 2007 in block Lakhanmajra, which is the field practice area attached to the department of Community Medicine, Pt. B.D. Sharma Post Graduate
Institute of Medical Sciences, Rohtak. The study subjects were school going children in the age group of 6-15 years. Out of total 16 Govt. schools existing in the block, two girls' schools and two boys' schools were randomly selected and all the students between 6-15 years of age, studying in class $1{ }^{\text {st }}$ to $10^{\text {th }}$ were included in the study. The students were divided in to three age groups: 6-10 years, 10-13 years and 13-15 years. All concerned Principals, teachers and students were briefed about the study. The students present on day of visit were included in the study. No follow up visits were done. The age of students was ascertained as per the school records. External examination of eyes was done by torch, lens and loupe.

Information was collected on a pretested semistructured schedule. After collection, the whole data was compiled; analyzed and appropriate statistical tests like simple proportions and chi-square $\left(?^{2}\right)$ test were applied.

\section{RESULTS}

$27.9 \%$ boys and $19.3 \%$ girls were suffering from one or more ocular infection. Squamous blepharitis was significantly associated with boys. All infections were more prevalent in boys as compared to girls. Table I shows sex 
wise distribution of eye problems. Table-II shows, most of the morbidities [24.3\%] were found in the age group 13-15 yrs.

\section{Figure 1}

Table 1: Sex wise distribution of eye problems

\begin{tabular}{|c|c|c|c|c|c|}
\hline \multirow[t]{2}{*}{ Ocular Infections } & \multicolumn{2}{|c|}{ Sex } & \multirow{2}{*}{$\begin{array}{c}\text { Total }[\%] \\
n=1265\end{array}$} & \multirow{2}{*}{$\begin{array}{c}\chi^{2} \\
\text { Value } \\
\text { (df } 1 \text { ) }\end{array}$} & \multirow{2}{*}{$\begin{array}{c}\text { p } \\
\text { Value }\end{array}$} \\
\hline & $\begin{array}{c}\text { Boys [\%] } \\
n^{-510}\end{array}$ & $\begin{array}{c}\text { Girls [\%] } \\
n^{-755}\end{array}$ & & & \\
\hline Squamous Blepharitis & $\begin{array}{c}75 \\
{[14.7]}\end{array}$ & $\begin{array}{c}81 \\
{[10.7]}\end{array}$ & $\begin{array}{c}156 \\
{[12.3]}\end{array}$ & 4.491 & $0.034^{+}$ \\
\hline Vernal Conjunctivitis & $\begin{array}{l}33 \\
{[65]}\end{array}$ & $\begin{array}{c}31 \\
{[4.1]}\end{array}$ & $\begin{array}{l}64 \\
{[5.1]}\end{array}$ & 335 & $>0.05$ \\
\hline Conjunctivitis & $\begin{array}{l}30 \\
{[59]}\end{array}$ & $\begin{array}{c}29 \\
{[38]}\end{array}$ & $\begin{array}{l}59 \\
{[4.7]}\end{array}$ & 2.65 & $>0.10$ \\
\hline Stye & $\begin{array}{c}4 \\
{[0.78]}\end{array}$ & $\begin{array}{c}5 \\
{[0.66]}\end{array}$ & $\begin{array}{c}9 \\
{[0.7]}\end{array}$ & 0064 & 080 \\
\hline Total & $\begin{array}{c}142 \\
{[279]}\end{array}$ & $\begin{array}{c}146 \\
{[19.3]}\end{array}$ & $\begin{array}{c}288 \\
{[22.8]}\end{array}$ & & \\
\hline
\end{tabular}

\section{Figure 2}

Table 2: Age wise distribution of eye problems

\begin{tabular}{|c|c|c|c|c|c|c|}
\hline \multirow[b]{2}{*}{$\begin{array}{c}\text { Ocular } \\
\text { Infections }\end{array}$} & \multicolumn{3}{|c|}{$\begin{array}{l}\text { Age Groups } \\
\text { [in years] }\end{array}$} & \multirow{2}{*}{$\begin{array}{c}\text { Total } \\
{[\%]} \\
n=1265 \\
{[\%]}\end{array}$} & \multirow{2}{*}{$\begin{array}{c}\chi^{2} \\
\text { Value } \\
\text { (df - 2) }\end{array}$} & \multirow{2}{*}{$\begin{array}{c}\text { p- } \\
\text { Value }\end{array}$} \\
\hline & $\begin{array}{c}6-10 \\
n=282 \\
{[\%]}\end{array}$ & $\begin{array}{c}10-13 \\
n=536 \\
{[\%]}\end{array}$ & $\begin{array}{c}13-15 \\
n=447 \\
{[\%]}\end{array}$ & & & \\
\hline $\begin{array}{l}\text { Squamous } \\
\text { Blepharitis }\end{array}$ & $\begin{array}{c}36 \\
{[12.8]}\end{array}$ & $\begin{array}{c}62 \\
{[115]}\end{array}$ & $\begin{array}{c}58 \\
{[13]}\end{array}$ & $\begin{array}{c}156 \\
{[123]}\end{array}$ & $0 A 96$ & 0.78 \\
\hline $\begin{array}{l}\text { Vermal } \\
\text { Conjunctivitis }\end{array}$ & $\begin{array}{l}14 \\
{[5.0]}\end{array}$ & $\begin{array}{c}30 \\
{[5.6]}\end{array}$ & $\begin{array}{c}20 \\
{[48]}\end{array}$ & $\begin{array}{c}64 \\
{[5.1]}\end{array}$ & 0.51 & 0.50 \\
\hline Conjumctivitis & $\begin{array}{c}10 \\
{[3.6]}\end{array}$ & $\begin{array}{c}23 \\
{[43]}\end{array}$ & $\begin{array}{l}26 \\
{[58]}\end{array}$ & $\begin{array}{l}59 \\
{[4.7]}\end{array}$ & 2.13 & $>0.10$ \\
\hline Stye & $\begin{array}{c}3 \\
{[1.1]}\end{array}$ & $\begin{array}{c}3 \\
{[0.6]}\end{array}$ & $\begin{array}{c}3 \\
{[0.7]}\end{array}$ & $\begin{array}{c}9 \\
{[0.7]}\end{array}$ & 0.690 & 0.70 \\
\hline Total & $\begin{array}{c}63 \\
{[22.5]}\end{array}$ & $\begin{array}{l}118 \\
{[22]}\end{array}$ & $\begin{array}{c}107 \\
{[243]}\end{array}$ & $\begin{array}{c}288 \\
{[228]}\end{array}$ & & \\
\hline
\end{tabular}

Table III shows, the prevalence of ocular infection was more in children $(27.4 \%)$ who did not wash their hand before meal as compared to those who $(22.3 \%)$ washed.

\section{Figure 3}

Table 3: Relation of eye problems with hand washing

\begin{tabular}{|c|c|c|c|c|c|}
\hline \multirow[b]{2}{*}{$\begin{array}{c}\text { Eye } \\
\text { Problems }\end{array}$} & \multicolumn{2}{|c|}{ Hand Washing } & \multirow[b]{2}{*}{$\begin{array}{c}\text { Total } \\
\mathrm{n}=1265 \\
{[\%]}\end{array}$} & \multirow[b]{2}{*}{$\begin{array}{c}\chi^{2} \text { Value } \\
\text { (df-1) }\end{array}$} & \multirow[b]{2}{*}{$\begin{array}{c}\mathbf{p} \\
\text { Value }\end{array}$} \\
\hline & $\begin{array}{c}\text { Yes } \\
\mathrm{n}=1130 \\
{[\%]}\end{array}$ & $\begin{array}{c}\text { No } \\
\mathrm{n}=135 \\
{[\%]}\end{array}$ & & & \\
\hline $\begin{array}{l}\text { Squamous } \\
\text { Blepharitis }\end{array}$ & $\begin{array}{c}136 \\
{[12.0]}\end{array}$ & $\begin{array}{c}20 \\
{[14.8]}\end{array}$ & $\begin{array}{c}156 \\
{[123]}\end{array}$ & 0.444 & 0598 \\
\hline $\begin{array}{l}\text { Vermal } \\
\text { Conjunctivitis }\end{array}$ & $\begin{array}{c}57 \\
{[5.0]}\end{array}$ & $\begin{array}{c}7 \\
{[5.2]}\end{array}$ & $\begin{array}{c}64 \\
{[5.1]}\end{array}$ & 0.67 & $>0.10$ \\
\hline Conjunctivitis & $\begin{array}{c}51 \\
{[4.7]}\end{array}$ & $\begin{array}{c}8 \\
{[59]}\end{array}$ & $\begin{array}{c}59 \\
{[4.7]}\end{array}$ & 0.016 & $>050$ \\
\hline Stye & $\begin{array}{c}7 \\
{[0.6]}\end{array}$ & $\begin{array}{c}2 \\
{[15]}\end{array}$ & $\begin{array}{c}9 \\
{[0.7]}\end{array}$ & 1.06 & 0.626 \\
\hline Total & $\begin{array}{c}251 \\
{[22.3]}\end{array}$ & $\begin{array}{c}37 \\
{[27.4]}\end{array}$ & $\begin{array}{c}288 \\
{[22.8]}\end{array}$ & & \\
\hline
\end{tabular}

The prevalence of ocular infection was more in children (23.3\%) who were using common towel as compared to those (17.3\%) using separate towel. Table IV shows relation of eye problems with usage of towel.

\section{Figure 4}

Table 4: Relation of eye problems with usage of towel

\begin{tabular}{|c|c|c|c|c|c|}
\hline \multirow[b]{2}{*}{ Eye Problems } & \multicolumn{2}{|c|}{ Separate Towel } & \multirow[b]{2}{*}{$\begin{array}{c}\text { Total } \\
\mathrm{n}=1265 \\
{[\%]}\end{array}$} & \multirow[b]{2}{*}{$\begin{array}{c}\chi^{2} \text { Value } \\
\text { (df-1) }\end{array}$} & \multirow[b]{2}{*}{$\begin{array}{c}\text { p } \\
\text { Value }\end{array}$} \\
\hline & $\begin{array}{c}\text { Yes } \\
\mathbf{n}=110 \\
{[\%]}\end{array}$ & $\begin{array}{c}\text { No } \\
\mathrm{n}=1155 \\
{[\%]}\end{array}$ & & & \\
\hline $\begin{array}{l}\text { Squamous } \\
\text { Blepharitis }\end{array}$ & $\begin{array}{c}12 \\
{[109]}\end{array}$ & $\begin{array}{l}144 \\
{[123]}\end{array}$ & $\begin{array}{l}156 \\
{[123]}\end{array}$ & 0.268 & 0.715 \\
\hline $\begin{array}{l}\text { Vernal } \\
\text { Conjunctivitis }\end{array}$ & $\begin{array}{c}4 \\
{[3.6]}\end{array}$ & $\begin{array}{c}60 \\
{[5.2]}\end{array}$ & $\begin{array}{c}64 \\
{[5.1]}\end{array}$ & 1.69 & $>0.10$ \\
\hline Conjunctivitis & $\begin{array}{c}3 \\
{[2.7]}\end{array}$ & $\begin{array}{l}56 \\
{[49]}\end{array}$ & $\begin{array}{c}59 \\
{[4.7]}\end{array}$ & 2.23 & $>050$ \\
\hline Stye & $\begin{array}{c}0 \\
\text { [0] }\end{array}$ & $\begin{array}{c}9 \\
{[0.8]}\end{array}$ & $\begin{array}{c}9 \\
{[0.7]}\end{array}$ & 0.84 & 1.00 \\
\hline Total & $\begin{array}{c}19 \\
{[173]}\end{array}$ & $\begin{array}{c}269 \\
{[23.3]}\end{array}$ & $\begin{array}{c}288 \\
{[22.8]}\end{array}$ & & \\
\hline
\end{tabular}

\section{DISCUSSION}

In this study the prevalence of ocular infections was found to be $22.8 \%$. This is higher than that reported by Kumar R et al (2004) ${ }_{2}$ and found to be $11.7 \%$ in rural and urban schools children of Delhi. The difference may be due to different study areas. Shreshtha et al (2006) ${ }_{3}$ observed 7.2\% prevalence of infective disorders in school children (5-16 yrs) of private schools of Kathmandu Valley. The difference was probably due to inclusion of private schools in the study. In the present study the prevalence of ocular infections were increased with increase in age groups. This finding is similar to that of Kumar R et al (2004) ${ }_{2}$.

In the present study, the prevalence of squamous blepharitis was found to be $12.3 \%$ which was much more than the prevalence found in the other studies i.e. Kumar $\mathrm{R}$ et al $(2004)_{2}$ observed 1\%, Trivedi et al (2006) ${ }_{4}$ observed $0.93 \%$.

In this study, the prevalence of conjunctivitis was found to be $4.7 \%$. Kumar R et al (2004) ${ }_{2}$ also observed $4.6 \%$ prevalence in urban and rural school children (5-14 yrs) of Delhi. Trivedi et al (2006) ${ }_{4}$ observed $5.1 \%$ prevalence in children (7-15 yrs) of urban and rural areas of Gujarat.

In the present study, the prevalence of vernal conjunctivitis was found to be $5.1 \%$. This is higher than that found in other studies like Shaffi et al (2005) ${ }_{5}$ who observed $0.7 \%$ prevalence. In the present study, the prevalence of stye was found to be $0.7 \%$. Kumar R et al (2004) ${ }_{2}$ observed $1.3 \%$ prevalence in school children (5-15 yrs) of Delhi (rural \& urban).

Thus, it can be concluded that ocular infections are quite prevalent in rural school children and health education 
regarding personal hygiene can play an important role in their prevention.

\section{References}

1. Ministry of Health \& Family Welfare, GOI, Community Ophthalmology Practice at Primary Care level. Study material for Medical Officers.2004. p.1.

2. Kumar R, Mehra M, Dobas P, Kamlesh, Raha R. A study of ocular infections amongst primary school children in Delhi. Indian J Commun Dis 2004; 36: 121-26.
3. Shrestha RK, Toshi MR, Ghisingh R, Pradhan P, Shahya $\mathrm{S}$, Rizyal A. Ocular morbidity among children studying in private school of Kathmandu Valley: A prospective cross sectional study. Nepal Med Coll J. 2006; 8: 43-6.

4. Trivedi V, Zalawadiya S, Bhatt JV, Pawar T, Kupmavat B. Prevalence of refractive errors in children of rural \& urban areas of Gujarat: A Population based study. Available from: http://openmed.nic.in/1933/01/bhatt 1.pdf.

5. Shaffi M, Bejiga A. Common eye diseases in children of rural community of Goro District, Central Ethiopia. Ethiop. J. Health Dev. 2005; 19: 148-52. 


\section{Author Information}

Seema Sharma

Department of Community Medicine, PGIMS

\section{BM Vashisht}

Department of Community Medicine, PGIMS

\section{Meenakshi Kalhan}

Department of Community Medicine, PGIMS

\section{Manish Goel}

Department of Community Medicine, PGIMS 